BULLETIN OF THE

AMERICAN MATHEMATICAL SOCIETY

Volume 80, Number 6, November 1974

\title{
UNITARY NILPOTENT GROUPS AND HERMITIAN $K$-THEORY. I
}

\author{
BY SYLVAIN E. CAPPELL ${ }^{1}$
}

Communicated by William Browder, February 19, 1974

This announcement computes the Wall surgery obstruction groups of amalgamated free products of finitely presented groups by using the new UNil functors introduced below. Special cases of these results [C4] were obtained as consequences of the splitting theorems of [C3]. The present results use the general results on manifold decomposition outlined in [C7]. Further applications to the study of manifolds and submanifolds, Poincaré duality spaces, diffeomorphism groups, and Novikov's conjecture [C8] will be presented elsewhere.

1. UNil of bimodules with involution. Let $R$ be a ring with unit and involution. Let $M$ be an $R$-bimodule with involution; i.e. $M$ is equipped with a homomorphism $x \rightarrow \bar{x}$ satisfying $\bar{x}=x,(\alpha x \beta)^{-}=\bar{\beta} \bar{x} \bar{\alpha}, x \in M$, $\alpha, \beta \in R$. Call $M$ hyperbolic if there is a decomposition of $R$-bimodules $M=N \oplus \bar{N}, \bar{N}=\{\bar{x} \mid x \in N \subset M\}$.

By a $(-1)^{k}$ Hermitian form over $M$ we mean a triple $(P, \lambda, \mu)$ where $P$ is a finitely generated free right $R$-module and $\lambda: P \times P \rightarrow M, \mu: P \rightarrow$ $M /\left\{x-(-1)^{k} \tilde{x} \mid x \in M\right\}$ satisfy:

(i) for $x \in P$ fixed, $y \rightarrow \lambda(x, y)$ is an $R$-homomorphism $P \rightarrow M$;

(ii) $\lambda(x, y)=(-1)^{k}(\lambda(y, x))^{-}, x, y \in P$;

(iii) $\lambda(x, x)=\mu(x)+(-1)^{k}(\mu(x))$ in $M, x \in P$;

(iv) $\mu(x+y)=\mu(x)+\mu(y)+\lambda(x, y), x, y \in P$;

(v) $\mu(x \alpha)=\bar{\alpha} \mu(x) \alpha, x \in P, \alpha \in P$.

Let $M_{1}$ and $M_{2}$ be $R$-bimodules with involution which are free left $R$-modules. A (resp; simple) $(-1)^{k}$ UNil form over $\left(M_{1}, M_{2}\right)$ is $C=$ $\left(P_{1}, \lambda_{1}, \mu_{1} ; P_{2}, \lambda_{2}, \mu_{2}\right)$ with $P_{2}=P_{1}^{*}$ and $\left(P_{i}, \lambda_{i}, \mu_{i}\right)$ a $(-1)^{k}$ Hermitian form over $M_{i}, i=1,2$, for which there exist finite filtrations of $R$-modules

$$
\begin{aligned}
& P_{1}=P_{1}^{0} \supset P_{1}^{1} \supset P_{1}^{2} \supset \cdots \supset P_{1}^{n}=0, \\
& P_{2}=P_{2}^{0} \supset P_{2}^{1} \supset P_{2}^{2} \supset \cdots \supset P_{2}^{m}=0
\end{aligned}
$$

so that, letting $\rho_{1}=P_{1} \rightarrow P_{2} \otimes_{R} M_{1}$ denote the adjoint of $\lambda_{1}$ and $\rho_{2}: P_{2} \rightarrow$ $P_{1} \otimes_{R} M_{2}$ denote the adjoint of $\lambda_{2}$,

$$
\rho_{1}\left(P_{1}^{i}\right) \subset P_{2}^{i+1} \otimes_{R} M_{1}, \quad \rho_{2}\left(P_{2}^{i}\right) \subset P_{1}^{i+1} \otimes_{R} M_{2}, \quad i \geqq 0
$$

AMS (MOS) subject classifications (1970). Primary 16A54, 20C05, 57A35, 57C35, 57D20, 57D40, 57D65, 18F25; Secondary 57D80, 18F30, 20H25, 20E30, 57B10, 16 A26.

1 The author is an A. P. Sloan fellow and was partially supported by an N.S.F. grant. 
(resp; and $\left(P_{1}, P_{2} ; \rho_{1}, \rho_{2}\right)$ represents the zero element in the group of nilpotent objects $\left(\widetilde{N} i l\left(R ; M_{1}, M_{2}\right)\right)$ defined in [W1]). Set $-C=\left(P_{1}\right.$, $\left.-\lambda_{1},-\mu_{1} ; P_{2},-\lambda_{2},-\mu_{2}\right)$. Call $C$ a (resp; simple) kernel if there are free summands $V_{i}$ of $P_{i}, i=1,2$, with $V_{2} \subset P_{2}=P_{1}^{*}$ the annihilator of $V_{1} \subset P_{1}$, and with $\left(\lambda_{i} \mid V_{i} \times V_{i}\right)$ and $\left(\mu_{i} \mid V_{i}\right)$ zero, $i=1,2$ (resp; and also for $\rho_{1}^{\prime}: P_{1} / V_{1} \rightarrow P_{2} / V_{2} \otimes_{R} M_{1}, \rho_{2}^{\prime}: P_{2} / V_{2} \rightarrow P_{1} / V_{1} \otimes_{R} M_{2}$, the induced maps, $\left(P_{1} / V_{1}, P_{2} / V_{2} ; \rho_{1}^{\prime}, \rho_{2}^{\prime}\right)$ represents zero in $\left.\left(\widetilde{N} i l\left(R ; M_{1}, M_{2}\right)\right)\right)$. Note that $C \oplus(-C)$ is a (resp; simple) kernel.

Introduce among the (resp; simple) $(-1)^{k}$ UNil forms over $\left(M_{1}, M_{2}\right)$ the equivalence relation generated by $A \sim B$ if $A \oplus(-B)$ is a (resp; simple) kernel. The equivalence classes form under the direct sum operation an abelian group denoted $\mathrm{UNil}_{2 k}^{h}\left(R ; M_{1}, M_{2}\right) \quad\left(\operatorname{resp} ; \mathrm{UNil}_{2 k}^{s}\left(R ; M_{1}, M_{2}\right)\right)$. Give $R\left[t, t^{-1}\right]$ the involution $\left(x t^{i}\right)^{-}=\bar{x} t^{-i}, x \in R$, and similarly introduce involutions on $M_{i} \otimes_{R} R\left[t, t^{-1}\right]$. Now define

$$
\begin{aligned}
& \mathrm{UNil}_{2 l_{-1}}^{h}\left(R ; M_{1}, M_{2}\right) \\
& \quad=\mathrm{UNil} l_{2 k}^{s}\left(R\left[t, t^{-1}\right] ; M_{1} \otimes_{R} R\left[t, t^{-1}\right], M_{2} \otimes_{R} R\left[t, t^{-1}\right]\right) / \mathrm{UNil} l_{2 k}^{s}\left(R ; M_{1}, M_{2}\right) .
\end{aligned}
$$

If $R$ is a regular ring, or even just coherent of finite global homological dimension, define

$$
\mathrm{UNil}_{2 k-1}^{s}\left(R ; M_{1}, M_{2}\right)=\mathrm{UNil}_{2 k-1}^{h}\left(R ; M_{1}, M_{2}\right) .
$$

Note the semiperiodicity $\mathrm{UNil}_{n}^{x}\left(R ; M_{1}, M_{2}\right) \cong \mathrm{UNil}_{n+2}^{x}\left(R ; M_{1}^{-}, M_{2}^{-}\right), x=s$ or $h$, where $M_{i}^{-}$is $M_{i}$ equipped with the involution $x \rightarrow-\bar{x}_{i}$.

2. Surgery groups of free products with amalgamation. Let $R \subset \Lambda_{1}$, $R \subset \Lambda_{2}$, be inclusions of rings with identity and involution. Assume $\Lambda_{i}$ has an $R$-bimodule with involution decomposition $\Lambda_{i}=R \oplus \hat{\Lambda}_{i}, \hat{\Lambda}_{i}$ a free left $R$-module. A $(-1)^{k}$ UNil form $\left(P_{1}, \lambda_{1}, \mu_{1} ; P_{2}, \lambda_{2}, \mu_{2}\right)$ over $\left(\hat{\Lambda}_{1}, \hat{\Lambda}_{2}\right)$ determines a $(-1)^{k}$ Hermitian form $(P, \lambda, \mu)$ over the free product with amalgamation ring $\Lambda_{1} *_{R} \Lambda_{2}$ with $P=\left(P_{1} \oplus P_{2}\right) \otimes_{R}\left(\Lambda_{1} *_{R} \Lambda_{2}\right)$ and with,

$$
\begin{gathered}
\lambda(x, y)=\langle x, y\rangle \quad \text { for } x \in P_{2}, y \in P_{1}\left(\text { recall } P_{2}=P_{1}^{*}\right), \\
\lambda(x, y)=\lambda_{i}(x, y) \quad \text { for } x, y \in P_{i}, \quad i=1,2, \\
\mu(x)=\mu_{i}(x) \text { for } x \in P_{i}, \quad i=1,2 .
\end{gathered}
$$

This construction induces for all $n$ a homomorphism $\operatorname{UNil}_{n}^{h}\left(R ; \hat{\Lambda} ; \hat{\Lambda}_{2}\right) \rightarrow$ $L_{n}^{h}\left(\Lambda_{1} *_{R} \Lambda_{2}\right)$, the Wall surgery group of $\Lambda_{1} *_{R} \Lambda_{2}$.

THEOREM 1. (i) The image of $\operatorname{UNil}_{n}^{h}\left(R ; \hat{\Lambda}, \hat{\Lambda}_{2}\right) \rightarrow L_{n}^{h}\left(\Lambda_{1} *_{R} \Lambda_{2}\right)$ is 2-primary.

(ii) If $\hat{\Lambda}_{1}$ and $\hat{\Lambda}_{2}$ are hyperbolic, or if 2 is invertible in $R$, the image of $\mathrm{UNil}_{n}^{h}\left(R ; \hat{\Lambda}_{1}, \hat{\Lambda}_{2}\right)$ in $L_{n}^{h}\left(\Lambda_{1} *_{R} \Lambda_{2}\right)$ is zero. 
Theorem 1 is proved algebraically by adapting the proof of $[\mathbf{C 3}$, Lemma II.10] and of Remark 2 at the end of [C3, \$2].

In the remainder of this paper, $R$ is a ring with $Z \subset R \subset Q$. The groups $H, G_{1}, G_{2}$ are finitely presented, $H \subset G_{1}$ and $H \subset G_{2}$. Moreover, $G_{1}$ and $G_{2}$ are assumed equipped with homomorphisms $\omega_{i}: G_{i} \rightarrow Z_{2}=\{ \pm 1\}$, with $\left(\omega_{1} \mid H\right)=\left(\omega_{2} \mid H\right)$; as usual, these determine the involution on $R\left[G_{i}\right]$ with $\bar{g}=\omega(g) g^{-1}, g \in G_{i} \subset R\left[G_{i}\right], i=1,2$. Let $R\left[\hat{G}_{i}\right]$ denote the $R[H]$ subbimodule with involution of $R\left[G_{i}\right]$ additively generated by $g \in\left\{G_{i}-H\right\}$.

THEOREM 2. The homomorphism $\operatorname{UNil}_{n}^{h}\left(R[H] ; R\left[\hat{G}_{1}\right], R\left[\hat{G}_{2}\right]\right) \rightarrow$ $L_{n}^{h}\left(R\left[G_{1} *_{H} G_{2}\right]\right)$ is a split monomorphism.

The splitting $\phi$ of this homomorphism is defined as follows. Realize $x \in L_{n}^{h}\left(R\left[G_{1} *_{H} G_{2}\right]\right)$, using [W2] for $R=Z$ and [CS] for general $R \subset Q$, by a normal cobordism of $1_{Y}$ to $f: W \rightarrow Y, Y$ a closed (n-1)-dimensional manifold, $n \geqq 6$, with $\pi_{1}(Y)=G_{1} *_{H} G_{2}, f$ an $R$-homotopy equivalence. Then from [C7], define $\phi(x)$ to be the splitting obstruction for $f$ along $X \subset Y$, where $\pi_{1} X=H$. Thus the action of $L_{n}^{h}\left(Z\left[G_{1} *_{H} G_{2}\right]\right)$ on $\mathscr{S}^{h}(Y)$, the set of $h$-cobordism classes of manifolds equipped with a homotopy equivalence to $Y$, restricts to a free action of $\mathrm{UNil}_{n}^{h}\left(Z[H] ; Z\left[\hat{G}_{1}\right], Z\left[\hat{G}_{2}\right]\right)$ on $\mathscr{S}^{h}(Y)$.

COROLLARY 3. $\mathrm{UNil}_{n}^{h}\left(R[H] ; R\left[\hat{G}_{1}\right], R\left[\hat{G}_{2}\right]\right)$ is a 2-primary group. If $\frac{1}{2} \in R$, it is zero.

Call a subgroup $K$ of a group $J$ square-root closed if $g^{2} \in K$ implies $g \in K$ for $g \in J$ [C3]. For example, if $K$ is normal in $J, K$ is square-root closed in $J$ if and only if $J / K$ has no elements of order 2. Any subgroup of a finite group of odd order is square-root closed. If $H$ is square-root closed in $G_{1}, Z\left[\hat{G}_{1}\right]$ is a hyperbolic $Z[H]$-bimodule with involution, hence:

Corollary 4. If $H$ is square-root closed in $G_{1}$ and $G_{2}$,

is zero.

$$
\mathrm{UNil}_{n}^{h}\left(R[H] ; R\left[\hat{G}_{1}\right], R\left[\hat{G}_{2}\right]\right)
$$

Thus, many results of [C3] can be obtained from [C7] using Theorem 1(ii). From the general splitting obstruction theory of [C7] we get:

THEOREM 5. (i) For $\Phi$ the quadrad of rings

$$
\begin{gathered}
R[H] \rightarrow R\left[G_{1}\right] \\
\downarrow \\
\downarrow \\
R\left[G_{2}\right] \rightarrow R\left[G_{1} *_{H} G_{2}\right]
\end{gathered}
$$

$$
\begin{aligned}
& L_{n}^{h}(\Phi)=\mathrm{UNil}_{n}^{h}(R[H]\left.; R\left[\hat{G}_{1}\right], R\left[\hat{G}_{2}\right]\right) \\
& \oplus H^{n-1}\left(Z_{2} ; \operatorname{Ker}\left(K_{0}(R[H]) \rightarrow K_{0}\left(R\left[G_{1}\right]\right) \oplus K_{0}\left(R\left[G_{2}\right]\right)\right)\right) .
\end{aligned}
$$


(ii) Let

$$
\begin{array}{r}
\hat{L}_{n}^{h}\left(R\left[G_{1} *_{H} G_{2}\right]\right) \\
\quad=\operatorname{CoKer}\left(\mathrm{UNil}{ }_{n}^{h}\left(R[H] ; R\left[\hat{G}_{1}\right], R\left[\hat{G}_{2}\right]\right) \rightarrow L_{n}^{h}\left(R\left[G_{1} *_{H} G_{2}\right]\right)\right) .
\end{array}
$$

Then if

$$
H^{i}\left(Z_{2} ; \operatorname{Ker}\left(K_{0}(R[H]) \rightarrow K_{0}\left(R\left[G_{1}\right]\right) \oplus K_{0}\left(R\left[G_{2}\right]\right)\right)\right)=0, \quad i \geqq 1,
$$

there is a long exact sequence

$$
\begin{aligned}
\cdots \rightarrow L_{n}^{h}(R[H]) & \rightarrow L_{n}^{h}\left(R\left[G_{1}\right]\right) \oplus L_{n}^{h}\left(R\left[G_{2}\right]\right) \\
& \rightarrow L_{n}^{h}\left(R\left[G_{1} *_{H} G_{2}\right]\right) \rightarrow L_{n-1}^{h}(R[H]) \rightarrow \cdots
\end{aligned}
$$

COROLlary 6. There is a long exact sequence for $x=h$ or for $x=s$, $\cdots \rightarrow L_{n}^{x}(R[H]) \otimes Z\left[\frac{1}{2}\right] \rightarrow\left(L_{n}^{x}\left(R\left[G_{1}\right]\right) \oplus L_{n}^{x}\left(R\left[G_{2}\right]\right)\right) \otimes Z\left[\frac{1}{2}\right]$

$$
\rightarrow L_{n}^{x}\left(R\left[G_{1} *_{H} G_{2}\right]\right) \otimes Z\left[\frac{1}{2}\right] \rightarrow L_{n-1}^{x}(R[H]) \otimes Z\left[\frac{1}{2}\right] \rightarrow \cdots
$$

\section{COROLlary 7. If}

$$
H^{i}\left(Z_{2} ; \operatorname{Ker}\left(K_{0}(R[H]) \rightarrow K_{0}\left(R\left[G_{1}\right]\right) \oplus K_{0}\left(R\left[G_{2}\right]\right)\right)\right)=0, \quad i \geqq 1,
$$

and if $\frac{1}{2} \in R$ or $H$ square-root closed in $G_{1}$ and $G_{2}$, there is a long exact sequence

$$
\begin{aligned}
\cdots \rightarrow L_{n}^{h}(R[H]) & \rightarrow L_{n}^{h}\left(R\left[G_{1}\right]\right) \oplus L_{n}^{h}\left(R\left[G_{2}\right]\right) \\
& \rightarrow L_{n}^{h}\left(R\left[G_{1} *_{H} G_{2}\right]\right) \rightarrow L_{n-1}^{h}(R[H]) \rightarrow \cdots
\end{aligned}
$$

Let $\mathscr{G}_{0}$ denote the smallest set of groups satisfying:

(i) $0 \in \mathscr{G}_{0}$;

(ii) if $H, G_{1}, G_{2} \in \mathscr{G}_{0}$, with $H \subset G_{i}, i=1,2$, then $G_{1} *_{H} G_{2} \in \mathscr{G}_{0}$;

(iii) if $H, J \in \mathscr{G}_{0}$ and $\xi_{i}: H \rightarrow J, i=1,2$, are monomorphisms, then $J *_{H}\{t\} \in \mathscr{G}_{0}$, where

$$
J *_{H}\{t\}=Z * J /\left\{t \xi_{1}(x) t^{-1} \xi_{2}(x)^{-1} \mid x \in H, t \text { the generator of } Z\right\} .
$$

From (iii), if $H \in \mathscr{G}_{0}$, then $Z \times H \in \mathscr{G}_{0}$. More generally, if $A, B \in \mathscr{G}_{0}$, then $A \times B \in \mathscr{G}_{0} . \mathscr{G}_{0}$ contains all torsion free finitely-generated one-relator groups and all fundamental groups of irreducible sufficiently large 3-manifolds.

Using [C2], [C4], a special case of the following result was proved in [Q]. From Corollary 3 we get

COROLlaRY 8. Let $L_{n}^{s}(G)$ denote the Wall surgery obstruction group for the simple homotopy equivalence problem for oriented manifolds with fundamental group $G$. If $G \in \mathscr{G}_{0}$,

$$
L_{n}^{s}(G) \otimes Z\left[\frac{1}{2}\right] \cong K O_{n}(K(G, 1)) \otimes Z\left[\frac{1}{2}\right]
$$


and

$$
L_{n}^{s}(G) \otimes Q \cong \bigoplus_{i \in Z} H_{n+4 i}(G ; Q) .
$$

This implies for a much larger set of groups than $\mathscr{G}_{0}$, Novikov's conjecture on homotopy invariance of the higher signatures [C8].

Problem. Let $\pi$ be the group of a locally flat $k$ not $S^{1} \subset S^{3}$; does the abelianization homomorphism $\pi \rightarrow Z$ induce an isomorphism of Wall groups [C1]? The present results show that $L_{n}^{s}(\pi)=L_{n}^{s}(Z) \oplus$ (a 2-primary group). For $\pi$ the group of a fibered knot, this 2-primary group is zero [C4].

3. Applications to Wall groups of fre products. For $0 \leqq m_{\imath} \leqq \infty$, $i=0, \pm 1, R\left(m_{-1}, m_{0}, m_{1}\right)$ denotes the free $R$-module on generators $x_{i}, y_{3}, z_{k}, 0<i \leqq m_{-1}, 0<j \leqq 2 m_{0}, 0<k \leqq m_{1}$, with involution determined by $\bar{x}_{i}=-x_{i}, \bar{z}_{k}=z_{k}, \bar{y}_{2 j}=y_{2 j-1}$. If $G$ is a group with $\omega: G \rightarrow Z_{2}=\{ \pm 1\}$ determining the involution on $R[G]$, then $R[\hat{G}] \cong R\left(m_{-1}, m_{0}, m_{1}\right)$, where $m_{0}$ is $\frac{1}{2}$ the number of $g \in G$ with $g^{2} \neq 1, m_{i}$ is the number of $g \in G$ satisfying $g^{2}=1, g \neq 1, \omega(g)=i$, for $i= \pm 1$.

Proposition 9. For $R$ a ring with $Z \subset R \subset Q$ :

(i)

$$
\begin{aligned}
\mathrm{UNil}_{n}^{h}(R ; R(a, b, c), R(d, e, f)) & \cong \mathrm{UNil}_{n}^{s}(R ; R(a, b, c), R(d, e, f)) \\
& \cong \mathrm{UNil}_{n+2}^{h}(R ; R(c, b, a), R(f, e, d))
\end{aligned}
$$

is 2-primary (2-torsion) for $n$ odd (even).

(ii) If $\frac{1}{2} \in R$, or $n$ odd and $m_{-1}+m_{1}+m_{-1}^{\prime}+m_{1}^{\prime}=0$, or $n=2 k$ and $m_{(-1)^{k+1}}+m_{(-1)^{k+1}}^{\prime}=0$, then

$$
\mathrm{UNil}_{n}^{h}\left(R ; R\left(m_{-1}, m_{0}, m_{1}\right), R\left(m_{-1}^{\prime}, m_{0}^{\prime}, m_{1}^{\prime}\right)\right)=0 .
$$

(iii) If $n=2 k, \quad \frac{1}{2} \notin R, \quad m_{(-1)^{k+1}}+m_{(-1)^{k+1}}^{\prime} \neq 0, \quad m_{-1}+m_{0}+m_{1} \neq 0$ and $m_{-1}^{\prime}+m_{0}^{\prime}+m_{1}^{\prime} \neq 0$, then

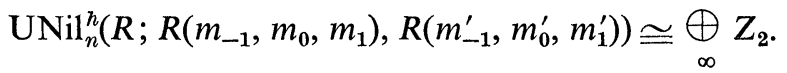

Let $\tilde{L}_{n}^{s}(G)$ denote the reduced surgery group, so that $L_{n}^{s}(G)=\tilde{L}_{n}^{s}(G) \oplus$ $L_{n}(0)$. The following extends results of [L], [C2], [C3], [C4], [C6].

THEOREM 10. Let $G_{1}$ and $G_{2}$ be finitely presented groups. Then

$$
L_{n}^{s}\left(G_{1} * G_{2}\right) \cong L_{n}(0) \oplus \tilde{L}_{n}^{s}\left(G_{1}\right) \oplus \tilde{L}_{n}^{s}\left(G_{2}\right) \oplus A,
$$


where $A$ is

(i) for $n=4 k$, zero;

(ii) for $n=4 k+1$ or $4 k+3$, zero if $G_{1}$ and $G_{2}$ have no elements of order 2 , and otherwise a 2-primary group,

(iii) for $n=4 k+2$, zero if and only if $G_{1}=0$, or $G_{2}=0$ or $G_{1}$ and $G_{2}$ have no elements of order 2; otherwise it is a vector space over $Z_{2}$ of infinite rank.

\section{BIBLIOGRAPHY}

[C1] Sylvain E. Cappell, Superspinning and knot complements, Topology of Manifolds, Markham, Chicago, Ill., 1970, pp. 358-383. MR 43 \#2711.

[C2] — , A splitting theorem for manifolds and surgery groups, Bull. Amer. Math. Soc. 77 (1971), 281-286. MR 44 \#2234.

[C3] — A splitting theorem for manifolds (to appear).

[C4] - Mayer-Vietoris sequences in Hermitian $K$-theory, Proc. Battelle $K$ Theory Conference, Lecture Notes in Math., vol. 343, Springer-Verlag, Berlin and New York, 1973, pp. 478-512.

[C5] —-, On connected sums of manifolds, Topology (to appear).

[C6] - Splitting obstructions for Hermitian forms and manifold with $Z_{2} \subset \pi_{1}$, Bull. Amer. Math. Soc. 79 (1973), 909-914.

[C7] - Manifolds with fundamental group a generalized free product. I, Bull. Amer. Math. Soc. (to appear).

[C8] - On the homotopy invariance of higher signatures (to appear).

[CS] Sylvain E. Cappell and Julius L. Shaneson, The codimension two placement problem and homology equivalent manifolds, Ann. of Math. (2) 99 (1974), 277-348.

[L] Ronnie Lee, Splitting a manifold into two parts, Mimeographed notes, Inst. Adv. Study, Princeton, N.J., 1969.

[Q] Frank Quinn, $B_{\left(\mathrm{TOP}_{n}\right)} \sim$ and the surgery obstruction, Bull. Amer. Math. Soc. 77 (1971), 596-600. MR 43 \#2718.

[W1] F. Waldhausen, Whitehead groups of generalized free products, (mimeographed preprint).

[W2] C. T. C. Wall, Surgery on compact manifolds, Academic Press, New York, 1970.

Department of Mathematics, Princeton University, Princeton, New Jersey 08540

Institut des Hautes Études Scientifiques, Paris, France

Current Address: Courant Institute of Mathematics, New York University, New York, New York 10012 